\title{
Bridging over troubled waters: the doubling time and histological subtypes of thymic epithelial tumors
}

\author{
Jumpei Kashima ${ }^{1,2}$, Yusuke Okuma ${ }^{3,4}$ \\ ${ }^{1}$ Department of Pathology, Tokyo Metropolitan Cancer and Infectious Diseases Center Komagome Hospital, Tokyo, Japan; ${ }^{2}$ Department of \\ Pathology, Graduate School of Medicine, The University of Tokyo, Tokyo, Japan; ${ }^{3}$ Department of Thoracic Oncology and Respiratory Medicine, \\ Tokyo Metropolitan Cancer and Infectious Diseases Center Komagome Hospital, Tokyo, Japan; ${ }^{4}$ Department of Thoracic Oncology, National \\ Cancer Center Hospital, Tokyo, Japan \\ Correspondence to: Yusuke Okuma. Department of Thoracic Oncology, National Cancer Center Hospital, Tsukiji 5-1-1, Chuo, Tokyo 104-0045, \\ Japan. Email: yokuma@ncc.go.jp. \\ Provenance and Peer Review: This article was commissioned and reviewed by the Section Editor Laura Chiara Guglielmetti (Cantonal Hospital \\ Winterthur, Kantonsspital Winterthur, Switzerland). \\ Comment on: Fukumoto K, Fukui T, Kawaguchi K, et al. The tumor doubling time is a useful parameter for predicting the histological type of thymic \\ epithelial tumors. Surg Today 2019;49:656-60.
}

Submitted Jan 28, 2020. Accepted for publication Feb 26, 2020.

doi: $10.21037 /$ jtd.2020.03.37

View this article at: http://dx.doi.org/10.21037/jtd.2020.03.37

Tumor doubling time (TDT) is a commonly-used indicator that reflects the radiographic growth rate of solid tumors and was proposed by Schwartz in 1961 (1). Three-dimensional volumetry has made precise estimation of solid tumors possible, which has led to accurate TDT calculation. Multiple studies have been conducted to determine the clinical and biological significance of TDT in several types of tumors. Lung cancer is one of the most thoroughly assessed tumors in terms of TDT. Not only prognosis but also stages and genetic mutations in the epidermal growth factor receptor have been reported to correlate with TDT $(2,3)$.

Comparisons of TDT and histology have been conducted as well. Lung squamous cell carcinoma has been reported to have a shorter TDT than lung adenocarcinoma (2-7), and the TDT of poorly differentiated tumors is shorter than that of well differentiated tumors (4). The TDT of the solid part of a lung tumor is significantly correlated with prognosis (2). Outside the thoracic cavity, clear cell renal cell carcinoma showed a correlation between TDT and histological grade (Fuhrman grade) (8). A multivariate analysis revealed that the molecular subtype and Ki-67 index confirmed by immunohistochemistry are significant factors related to TDT (9).

Histopathological diagnosis is inevitable for the initiation of treatment of solid malignancies, including surgery, radiotherapy, and chemotherapy, except for limited cases in an emergency. Histological subtype can be a key factor in the selection of chemotherapy as well. For example, bevacizumab and pemetrexed are not used for lung squamous cell carcinoma. Therefore, the clinical significance of speculated histological subtype by radiography has been limited thus far. In some situations, however, such as the treatment of patients who cannot undergo or have not yet undergone surgery or biopsy, the estimation of histology by radiological images may be useful in considering the frequency of follow-up and further treatment strategies. Moreover, the correlation between histological subtypes and radiological features has not been well studied in rare malignancies.

Fukumoto et al. assessed the TDT of thymic epithelial tumors that were surgically resected (10). The histological subtype of the tumor was graded as a low-risk thymoma (LRT) (type A, AB, and B1), high-risk thymoma (HRT) (B2 and B3), or thymic carcinoma (TC). In total, 96 out of 130 patients showed tumor growth, and the TDT of LRT and HRT significantly differed from TC. A receiver operating characteristic (ROC) curve analysis showed an area under the curve of 0.866 , and the sensitivity and specificity were $83.8 \%$ and $82.1 \%$, respectively, when the threshold was set 
Table 1 Tumor doubling time of thymic epithelial tumors assessed in the previous studies

\begin{tabular}{|c|c|c|c|c|c|c|c|}
\hline Study & \multicolumn{3}{|c|}{$\mathrm{n}$} & \multicolumn{3}{|c|}{ Median TDT [range], days } & $\begin{array}{l}\text { Threshold determined with } \\
\text { a ROC curve (Sn, Sp), days }\end{array}$ \\
\hline Jeong (12) & 15 & 26 & 9 & $703.6^{\star}[286.7-1,855.9]$ & $412.1^{*}[130.9-716.9]$ & $146.3^{*}[68.9-448.3]$ & $222.6^{*}(90.2 \%, 77.8 \%)$ \\
\hline Choe (13) & 24 & 5 & 4 & $436[62-1,964]$ & $381[58-1,282]$ & 189 [48-260] & NA \\
\hline Fukumoto (10) & 43 & 25 & 28 & $607[170-6,188]$ & 459 [116-3,005] & 205 [43-725] & $313(83.8 \%, 82.1 \%)$ \\
\hline
\end{tabular}

*, TDT calculated with the longest diameter are shown here. LRT, low-risk thymoma; HRT, high-risk thymoma; TC, thymic carcinoma; TDT, tumor doubling time; ROC, receiver operating characteristic; Sn, sensitivity; Sp, specificity; NA, not available.

at 313 days to discriminate thymoma from TC.

Since both thymoma and TC occasionally show invasive growth and metastases, they are categorized as thymic malignancies. A study by Fukumoto et al. is significant in terms of showing the rate of growth of relatively rare malignancies from as many as 96 patients (10). The results, which demonstrated that the majority of thymomas enlarged during follow-up and some of them grew as rapidly as TC, reminds us that both subtypes are malignant, even though the TDT of TC is shorter than that of thymoma as a whole. In addition, 25 out of $130(19 \%)$ thymic epithelial tumors were stable in size, and nine tumors, including TC, even regressed. This phenomenon is not specific to thymic epithelial tumors, as the studies on lung cancer and renal cell carcinoma mentioned above also showed shrinking tumors $(\mathrm{TDT}<0)(6,11)$. Comparing radiographic images and histology may contribute to revealing the mechanism of and predicting factors for spontaneous tumor shrinkage.

To the best of our knowledge, three studies that examined the TDT of thymic epithelial tumors have been published $(10,12,13)$ (Table 1). These three studies employed calculation methods based on the theory proposed by Schwartz (1) and obtained consistent results. Jeong et al. also adopted three-dimensional volumetry methods. The formula for TDT calculation adopted by Choe et al. uses both the maximum tumor diameter and the diameter that is perpendicular to it (13). A study by Fukumoto et al. enrolled the most patients, especially those with TC.

We roughly integrated the results from these three studies using median and interquartile range of the TDT of the three groups of tumors with a random effects model (Figure 1), and statistical calculations were conducted using R version 3.6.1 (The R Foundation for Statistical Computing, Vienna, Austria). Both LRT and HRT had a significantly longer TDT than TC [mean difference (MD) of TDT: 366.59 days; $\mathrm{P}<0.001$ and 261.65 days; $\mathrm{P}<0.001$, respectively]. In contrast, the difference between LRT and HRT was comparatively small (MD: 105.15 days; $\mathrm{P}=0.079$ ).

The distribution of the TDT of each thymoma subtype was noted only in the study by Choe et al. (13), which showed some outstanding outliers in type B1 thymoma. The other two studies also indicated a wide range of TDT values for LRT (Table 1). In addition, type B3 thymoma seemed to be separated into those with a long TDT and those with a short TDT (13). These results imply that there is a heterogeneous population in certain histological subtypes of thymoma in terms of proliferation activity. Clinical findings, such as the initial tumor diameter and stage, were not associated with the TDT $(10,12,13)$, but pathological factors other than subtype, including the Ki-67 index, may have the potential to further predict aggressive tumor growth. Moreover, TDT and prognosis may even be correlated, considering the link between prognosis and histological subgroup.

Radiological images play a key role, especially in situations in which biopsy is not feasible. If malignancy is highly suspected, surgical intervention is considered, and its risks and benefits are compared. All the studies on the TDT of thymic epithelial tumors focused on patients who underwent surgical resection. This aspect could have biased patient selection. Extremely slow-growing tumors might not have been resected and excluded from the studies. In contrast, an enlarging tumor at an exceptional rate would more likely be resected and included in the investigations.

These studies were retrospective, and the frequencies and intervals between image documentation were uneven. TDT can be computed by a formula utilizing the maximum tumor diameters at two observational points, but the background theory for this calculation is based on the continuous exponential growth of tumors. Multiple observations would enable us to capture more precise fluctuation in tumor growth. 


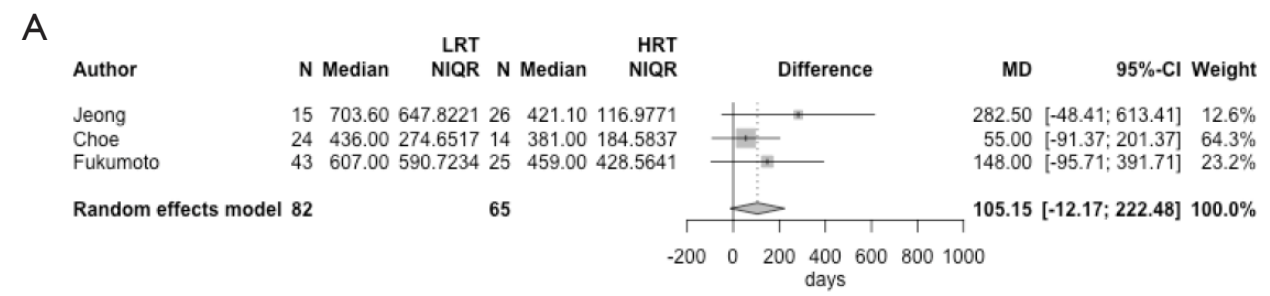

B

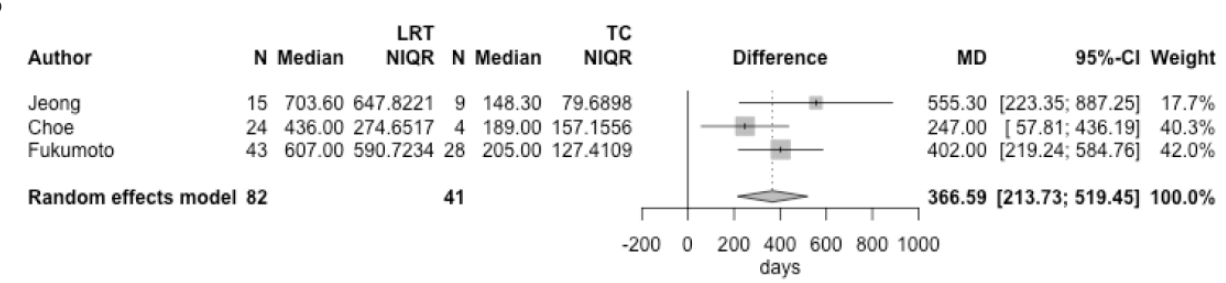

C

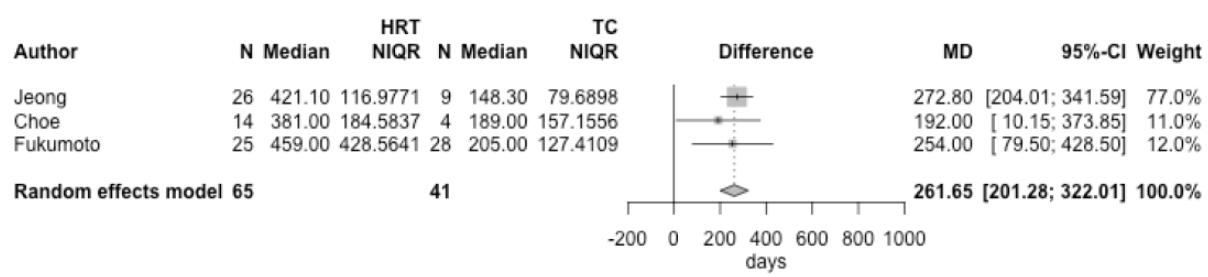

Figure 1 Summary of the difference among the previously assessed TDTs of thymic epithelial tumors $(10,12,13)$. (A) LRT vs. HRT; (B) LRT vs. TC; (C) HRT vs. TC. TDTs, tumor doubling times; LRT, low-risk thymoma; HRT, high-risk thymoma; TC, thymic carcinoma; $\mathrm{NIQR}$, normalized interquartile range; $\mathrm{MD}$, mean difference; $\mathrm{CI}$, confidence interval.

As noted above, histopathological diagnosis is essential in the initiation of treatment for malignancies. TDT, however, provides time-lapse information, which is hard to obtain from histology. Integrated analyses of histology, radiological images, and clinical information by machine learning have the potential to create models that predict the exact prognosis. Treatment for thymic epithelial tumors are still being developed. Bridging radiological images and pathological subtyping may bring about precision medicine for rare malignancies.

\section{Acknowledgments}

The authors thank Enago (www.enago.jp) for the English language review.

Funding: None.

\section{Footnote}

Conflicts of Interest: Both authors have completed the ICMJE uniform disclosure form (available at http://dx.doi. org/10.21037/jtd.2020.03.37). The authors have no conflicts of interest to declare.

Ethical Statement: The authors are accountable for all aspects of the work in ensuring that questions related to the accuracy or integrity of any part of the work are appropriately investigated and resolved.

Open Access Statement: This is an Open Access article distributed in accordance with the Creative Commons Attribution-NonCommercial-NoDerivs 4.0 International License (CC BY-NC-ND 4.0), which permits the noncommercial replication and distribution of the article with the strict proviso that no changes or edits are made and the original work is properly cited (including links to both the formal publication through the relevant DOI and the license). See: https://creativecommons.org/licenses/by-nc-nd/4.0/.

\section{References}

1. Schwartz M. A biomathematical approach to clinical tumor 
growth. Cancer 1961;14:1272-94.

2. Miura K, Hamanaka K, Koizumi T, et al. Solid component tumor doubling time is a prognostic factor in non-small cell lung cancer patients. J Cardiothorac Surg 2019;14:57.

3. Nakamura R, Inage Y, Tobita R, et al. Epidermal growth factor receptor mutations: effect on volume doubling time of non-small-cell lung cancer patients. J Thorac Oncol 2014;9:1340-4.

4. Mackintosh JA, Marshall HM, Yang IA, et al. A retrospective study of volume doubling time in surgically resected non-small cell lung cancer. Respirology 2014;19:755-62.

5. Obayashi K, Shimizu K, Nakazawa S, et al. The impact of histology and ground-glass opacity component on volume doubling time in primary lung cancer. J Thorac Dis 2018;10:5428-34.

6. Honda O, Johkoh T, Sekiguchi J, et al. Doubling time of lung cancer determined using three-dimensional volumetric software: comparison of squamous cell carcinoma and adenocarcinoma. Lung Cancer 2009;66:211-7.

7. Lindell RM, Hartman TE, Swensen SJ, et al. Five-year lung cancer screening experience: CT appearance, growth rate, location, and histologic features of 61 lung cancers.

Cite this article as: Kashima J, Okuma Y. Bridging over troubled waters: the doubling time and histological subtypes of thymic epithelial tumors. J Thorac Dis 2020;12(7):3886-3889. doi: $10.21037 /$ jtd. 2020.03 .37
Radiology 2007;242:555-62.

8. Zhang L, Yin W, Yao L, et al. Growth pattern of clear cell renal cell carcinoma in patients with delayed surgical intervention: fast growth rate correlates with high grade and may result in poor prognosis. Biomed Res Int 2015;2015:598134.

9. Zhang S, Ding Y, Zhou Q, et al. Correlation factors analysis of breast cancer tumor volume doubling time measured by 3D-ultrasound. Med Sci Monit 2017;23:3147-53.

10. Fukumoto K, Fukui T, Kawaguchi K, et al. The tumor doubling time is a useful parameter for predicting the histological type of thymic epithelial tumors. Surg Today 2019;49:656-60.

11. Zhang J, Kang SK, Wang L, et al. Distribution of renal tumor growth rates determined by using serial volumetric CT measurements. Radiology 2009;250:137-44.

12. Jeong DY, Lee KS, Chung MJ, et al. JOURNAL CLUB: doubling time of thymic epithelial tumors correlates with World Health Organization histopathologic classification. AJR Am J Roentgenol 2017;209:W202-10.

13. Choe J, Lee SM, Lim S, et al. Doubling time of thymic epithelial tumours on CT: correlation with histological subtype. Eur Radiol 2017;27:4030-6. 